\title{
A Rare Case of Rheumatoid Arthritis with Tocilizumab- induced Intestinal Mucosal Injury
}

\author{
Akito Ohkubo ${ }^{1}$, Takashi Osoegawa ${ }^{1}$, Naohiko Harada ${ }^{1}$, Yoichiro Iboshi ${ }^{1}$, Yorinobu Sumida ${ }^{1}$, \\ Makoto Nakamuta ${ }^{1}$, Eiichi Suematsu ${ }^{2}$, Hiroyuki Kobayashi ${ }^{3}$ and Eikichi Ihara ${ }^{4}$
}

\begin{abstract}
:
Intestinal mucosal injury that develops as a complication of tocilizumab (TCZ) is usually associated with diverticulosis. We herein report a rare case of TCZ-induced intestinal mucosal injury in the absence of diverticulosis. A 74-year-old woman suffering from rheumatoid arthritis started taking TCZ. Six months later, she complained of hematochezia and abdominal pain. Colonoscopy revealed multiple ulcers spreading from the cecum to the transverse colon but no diverticulosis. These lesions were cured at three months after the discontinuation of TCZ. We should consider TCZ as a risk factor for intestinal mucosal injury, even if patients have no history of intestinal disease associated with diverticulosis.
\end{abstract}

Key words: Tocilizumab, IL-6, enteritis, rheumatoid arthritis

(Intern Med Advance Publication)

(DOI: 10.2169/internalmedicine.8031-21)

\section{Introduction}

Interleukin (IL)-6 is a major pleiotropic cytokine that regulates inflammatory and immunological responses. (1) Overproduction of IL-6 is involved in the underlying pathogenesis of certain autoinflammatory diseases, such as rheumatoid arthritis (RA). $(1,2)$ High levels of IL-6 were detected in the peripheral blood and synovial fluid of patients with RA, (3) suggesting that IL-6 might be a good therapeutic target for RA. $(4,5)$

Tocilizumab (TCZ) is a humanized anti-human IL-6 receptor monoclonal antibody that inhibits the binding of IL-6 to the membrane-bound IL-6 receptor. Indeed, TCZ exerts therapeutic effects for RA as well as other autoinflammatory diseases, including Castleman's disease, juvenile idiopathic arthritis, and Takayasu arteritis. $(4,5)$ Recently, TCZ was used as a treatment option for severe COVID-19 patients at risk of a cytokine storm. Therefore, TCZ might be used more frequently in clinical settings than before.

However, IL-6 plays a role in intestinal proliferation and wound repair, and the blockade of IL- 6 by TCZ carries a risk of causing intestinal mucosal injury. (6) Indeed, intestinal perforations reportedly developed as a complication of TCZ treatment, although most of these were associated with concomitant diverticulosis, another risk factor for perforation. (7-9) The underlying pathogenesis of TCZ-induced intestinal mucosal injury therefore remains to be determined.

We herein report a rare case of RA causing intestinal mucosal injury as a complication of TCZ in the absence of diverticulosis.

\section{Case Report}

A 74-year-old woman diagnosed with RA in January 2014 was administered a combined treatment of prednisolone, bucillamine, loxoprofen, and methotrexate. Because her clinical condition could not be satisfactorily improved by conventional treatment, she was referred to our hospital.

Annual colonoscopy revealed neither ulcers nor diverticulosis in April 2014. She started to receive treatment with the molecular-targeted drug TCZ (162 mg) biweekly in April

${ }^{1}$ Department of Gastroenterology, Clinical Research Institute, National Hospital Organization Kyushu Medical Center, Japan, ${ }^{2}$ Department of Rheumatology, Clinical Research Institute, National Hospital Organization Kyushu Medical Center, Japan, ${ }^{3}$ Department of Gastroenterology, Fukuoka Sanno Hospital, Japan and ${ }^{4}$ Department of Medicine and Bioregulatory Science, Graduate School of Medical Sciences, Kyushu University, Japan

Received: May 26, 2021; Accepted: August 1, 2021; Advance Publication by J-STAGE: September 18, 2021

Correspondence to Dr. Naohiko Harada, naoharadan@gmail.com 


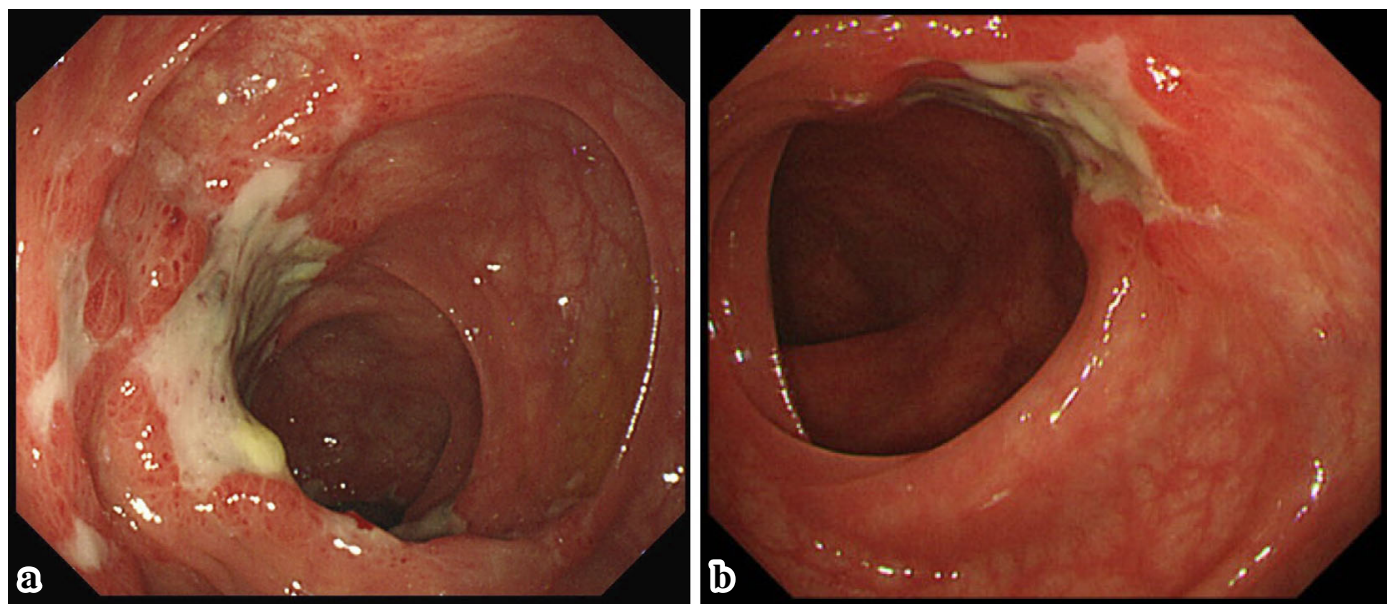

Figure 1. Colonoscopy showing multiple irregular ulcers from the cecum to the transverse colon in October 2015. (a) Ascending colon, (b) transverse colon.

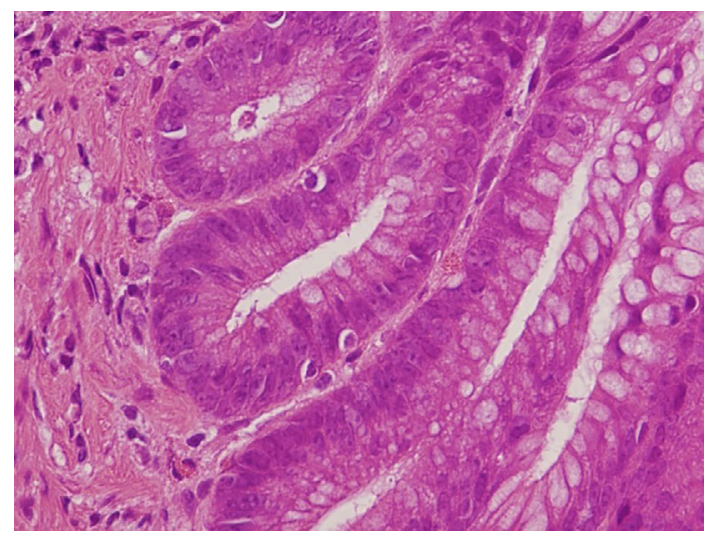

Figure 2. Histopathological image showing few apoptoses in the crypt epithelium.

2015, and her symptoms improved. In October, six months after the initiation of TCZ, she complained of hematochezia and abdominal pain. Urgent colonoscopy revealed multiple irregular ulcers spreading from the cecum to the transverse colon but no evidence of diverticulosis (Fig. 1). The pathological results of biopsy samples showed a few apoptoses in the crypt epithelium (Fig. 2). No specific findings were obtained from bacterial cultures of colonic mucosa, and no positive findings were obtained for suspected infection by tuberculosis or cytomegalovirus. Blood tests showed a mildly elevated inflammation index with a white blood cell count and C-reactive protein level of $12,700 / \mu \mathrm{L}$ and 0.08 $\mathrm{mg} / \mathrm{dL}$, respectively, but no other positive findings by an interferon-gamma release assay or observations related to cytomegalovirus pp-65 antigen.

In addition to TCZ, she was taking regular medications of prednisolone, methotrexate, loxoprofen, foliamin, lansoprazole, rebamipide, levothyroxine sodium hydrate, rosuvastatin calcium, ezetimibe, and fluconazole. The intestinal mucosal injury was suspected of having developed as a complication of TCZ.

As a result, the discontinuation of TCZ treatment im-

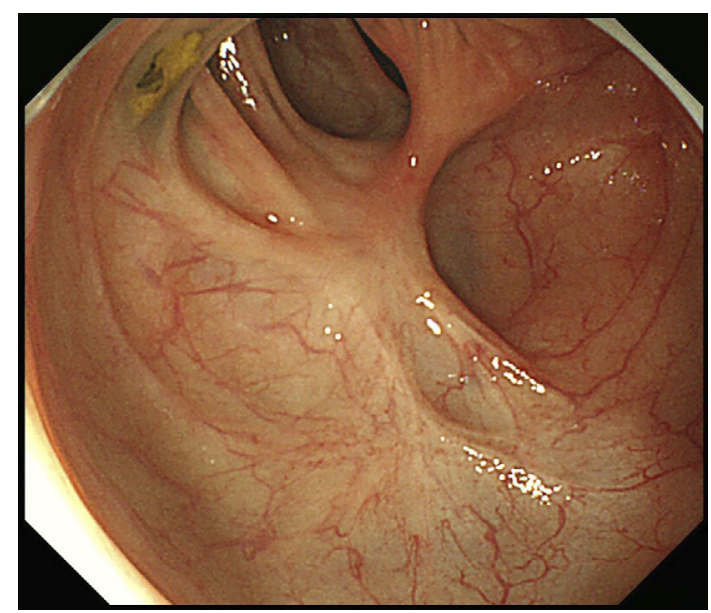

Figure 3. Ascending colon. Colonoscopy showing multiple scars in January 2016.

proved hematochezia and abdominal pain in the patient. Follow-up colonoscopy at one month after the discontinuation of TCZ in November 2015 revealed that multiple ulcers had healed, and all of the ulcers had completely cured, as reflected by scarring noted in January 2016 (Fig. 3). She has not complained of hematochezia or abdominal pain since January 2016. In February 2016, she was administered tofacitinib instead of TCZ, and RA did not relapse.

\section{Discussion}

IL-6 is an important cytokine that contributes to physiological and/or pathological responses, such as the regulation of inflammatory and immunological responses, as well as the activation of osteoclasts and angiogenesis. (1) IL-6 is involved in the underlying pathogenesis of autoinflammatory diseases, including RA, juvenile idiopathic arthritis, Takayasu's arteritis, giant cell arteritis, and Castleman's disease. $(4,5)$

TCZ, a recombinant humanized monoclonal antibody di- 
rected against the human IL-6 receptor, is used to treat autoinflammatory diseases, such as RA. (1-3) Although various adverse events associated with TCZ treatment have been reported, (10) intestinal adverse events are rare. Furthermore, most of them have been cases of intestinal perforation associated with diverticulosis. (7-9) Prior diverticulitis was reported to be a risk factor for intestinal perforation associated with IL-6 inhibition. (7) The mechanism underlying intestinal perforation was reported to involve TCZ administration masking abdominal pain and CRP elevation and inhibiting recovery from intestinal injuries caused by diverticulitis. (11) We encountered a rare case of RA with TCZinduced intestinal injury that was not associated with diverticulosis.

Few cases of TCZ-induced intestinal mucosal injury have been reported to date. $(12,13)$ The characteristics of the lesions are unknown, and the diagnostic criteria for TCZinduced intestinal mucosal injury have not been reported. Therefore, its diagnosis is made by the exclusion of other potential diseases. In the present case, we diagnosed our patient with TCZ-induced intestinal mucosal injury based on the following: i) no ulcer lesions were detected by colonoscopy before TCZ administration, ii) multiple colonic ulcers were observed at six months after the initiation of TCZ therapy, iii) these lesions were healed at one month and completely cured at three months after the discontinuation of TCZ, iv) no specific findings were obtained from the bacterial culture of colonic mucosa or indicated infection by tuberculosis and cytomegalovirus, v) the doses of loxoprofen and prednisolone were unchanged before and after the occurrence of the symptoms, and vi) she had no sudden-onset abdominal symptoms, such as ischemic colitis, and the endoscopic findings differed completely from those of ischemic colitis.

The induction of intestinal mucosal injury by the readministration of TCZ would have strongly supported a diagnosis of TCZ-induced intestinal mucosal injury; however, this could not be attempted in the present case for ethical reasons. Of note, one case report showed that TCZ-induced intestinal mucosal injury was induced by the incidental readministration of TCZ. (12)

The pathological mechanism underlying TCZ-induced intestinal ulcers remains unclear. TCZ-induced intestinal mucosal injury is rare and is thought to occur under special conditions. Intestinal perforations as a complication of TCZ treatment were associated with concomitant diverticulosis. Furthermore, most of these patients were concurrently prescribed non-steroidal anti-inflammatory drugs (NSAIDs) and/or long-term corticosteroids. (9) Indeed, both the present case and one reported by Iwasa et al. had no diverticulosis but were taking celecoxib and loxoprofen. (12) Basic studies have shown that IL-6 is upregulated soon after intestinal injury and plays an important role in intestinal proliferation and wound repair. (6) TCZ, a monoclonal antibody against the IL-6 receptor, might delay wound repair and enhance NSAID-induced intestinal injury. Indeed, discrete ulcers in the present case were similar to those observed in NSAIDinduced intestinal injury, and typical apoptotic features associated with NSAID-induced intestinal injury were seen in biopsy samples. More recently, it was reported that COVID19 patients treated with TCZ developed ulcerative lesions spreading from the ileum to the ascending colon. (13) Systemic hypoperfusion and the use of vasopressors in combination with a wound repair function potentially impaired by TCZ might be associated with intestinal mucosal injury. Further clinical studies, such as case-control studies, will be required to determine the clinical risk factors associated with TCZ-induced intestinal mucosal injury.

In conclusion, we experienced a rare case of RA with TCZ-induced intestinal mucosal injury. We should consider TCZ to carry a risk of inducing intestinal mucosal injury, even if patients have no history of intestinal diseases, including diverticulosis.

\section{The authors state that they have no Conflict of Interest (COI).}

\section{Authors' contributions}

A.O.: Study concept, acquisition of data, drafting and revision of the manuscript; T.O.: Study concept, acquisition of data; N.H.: Study concept, acquisition of data, drafting and revision of the manuscript; Y.I.: Drafting and revision of the manuscript; Y.S.: Drafting and revision of the manuscript; M.N.: Drafting and revision of the manuscript; E.S.: Study concept and design, acquisition of data; H.K.: Study concept and design, acquisition of data; E.I.: Drafting and revision of the manuscript. All authors read and approved the final manuscript.

\section{References}

1. Kishimoto T. Interleukin-6: from basic science to medicine -40 years in immunology. Annu Rev Immunol 23: 1-21, 2005.

2. Nishimoto N. Clinical studies in patient with Castleman's disease, Crohn's disease, and rheumatoid arthritis in Japan. Clin Rev Allergy Immunol 28: 221-230, 2005.

3. Houssiau FA, Devogelaer JP, Van Damme J, de Deuxchaisnes CN, Van Snick J. Interleukin-6 in synovial fluid and serum of patients with rheumatoid arthritis and other inflammatory arthritis. Arthritis Rheum 31: 784-788, 1988.

4. Nishimoto N, Kishimoto T. Humanized antihuman IL-6 receptor antibody, tocilizumab. Handb Exp Pharmacol 181: 151-160, 2008.

5. Ogata A, Kato Y, Higa S, Yoshizaki K. IL-6 inhibitor for the treatment of rheumatoid arthritis: A comprehensive review. Modern Rheumatology 29: 258-267, 2019.

6. Kuhn KA, Manieri NA, Liu TC, Stappenbeck TS. IL-6 stimulates intestinal epithelial proliferation and repair after injury. PLOS ONE 9: e114195, 2014.

7. Strangfeld A, Richter A, Siegmund B, et al. Risk for lower intestinal perforations in patients with rheumatoid arthritis treated with tocilizumab in comparison to treatment with other biologic or conventional synthetic DMARDs. Ann Rheuma Dis 76: 504-510, 2017.

8. Xie F, Yun H, Bernatsky S, et al. Risk for gastrointestinal perforation among rheumatoid arthritis patients receiving tofacitinib, tocilizumab, or other biologic treatments. Arthritis Rheumatology 68: 2612-2617, 2016.

9. Gout T, Östör AJK, Nisar MK. Lower gastrointestinal perforation 
in rheumatoid arthritis patients treated with conventional DMARDs of tocilizumab: a systematic literature review. Clin Rheumatol 30: 1471, 2011.

10. Nishimoto N, Ito K, Takagi N. Safety and efficacy profiles of tocilizumab monotherapy in Japanese patients with rheumatoid arthritis: meta-analysis of six initial trials and five long-term extensions. Mod Rheumatol 20: 222-232, 2010.

11. Ogata A, Kato Y, Higa S, Yoshizaki K. IL-6 inhibitor for the treatment of rheumatoid arthritis: A comprehensive review. Mod Rheumatol 29: 258-267, 2019.
12. Iwasa $\mathrm{T}$, Nakamura $\mathrm{K}$, Ogino $\mathrm{H}$, et al. Multiple ulcers in the small and large intestines occurred during tocilizumab therapy for rheumatoid arthritis. Endoscopy 43: 70-72, 2011.

13. Bruce-Hickman D, Sajeed SM, Pang YH, et al. Bowel ulceration following tocilizumab administration in a COVID-19 patient. BMJ Open Gastro 7: e000484, 2020.

The Internal Medicine is an Open Access journal distributed under the Creative Commons Attribution-NonCommercial-NoDerivatives 4.0 International License. To view the details of this license, please visit (https://creativecommons.org/licenses/ by-nc-nd/4.0/).

(C) The Japanese Society of Internal Medicine Intern Med Advance Publication 